Bioinspired, Biomimetic and Nanobiomaterials Volume 2 Issue BBN4

Biofunctionalized nanofibrous membranes mimicking carnivorous plants

Homaeigohar, Disci-Zayed, Dai and Elbahri
Pages 186-193 http://dx.doi.org/10.1680/bbn. 13.00006 Themed Issue Research Paper Received 21/02/2013 i

ce

\title{
Biofunctionalized nanofibrous membranes mimicking carnivorous plants
}

1 Shahin Homaeigohar Dr. Ing.

Helmholtz-Zentrum Geesthacht, Institute of Polymer Research, Nanochemistry and Nanoengineering group, Geesthacht, Germany

2 Duygu Disci-Zayed MSC

Nanochemistry and Nanoengineering group, Institute for Materials Science, Faculty of Engineering, University of Kiel, Kaiserstrasse 2, Kiel, Germany

3 Tianhe Dai Dr. Ing

Helmholtz-Zentrum Geesthacht, Institute of Polymer Research, Nanochemistry and Nanoengineering group, Geesthacht, Germany

\section{Mady Elbahri Prof. Dr. Ing.*}

Helmholtz-Zentrum Geesthacht, Institute of Polymer Research, Nanochemistry and Nanoengineering group, Geesthacht, Germany

Nanochemistry and Nanoengineering group, Institute for Materials Science, Faculty of Engineering, University of Kiel, Kaiserstrasse 2, Kiel, Germany
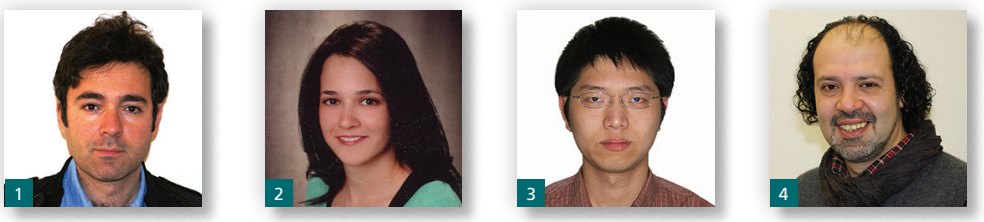

A novel biofunctionalized nanofibrous membrane is developed through immobilization of protein ligands on the surface of nanofibers. The biofunctionalization not only enhances the membrane's structural properties including mechanical and thermal ones but also makes the membrane capable to separate nanoparticles and biomolecules much smaller than the pore size from water efficiently. Upon contact with water, the conformational change of the protein immobilized leads to its swelling, thereby an enlarged functional surface area and a higher steric hindrance capturing the filtrates. In case of filtration of a plasmonic nanoparticle containing suspension, decoration of the membrane with the plasmonic nanoparticles forms a smart bionanocomposite biosensor for detection of protein denaturation.

\section{Introduction}

Membrane technology for water treatment is steadily gaining very high importance worldwide. This is primarily due to water pollution and dwindling fresh water supplies leading to water scarcity. Water quality has to be controlled to ensure a safer environment by implementing efficient technologies such as advanced membranes offering more output with less input, that is, efficient energy saving membranes. Electrospun nanofibrous membranes (ENMs) that have the potential to be used as advanced membrane systems will be able to remove pollutants from the environment at lower energy and hence cost. ${ }^{1}$ Energy saving by ENMs derives from their high interconnected porosity leading to a very high permeability. ${ }^{2}$ Despite an extraordinary permeability, ENMs suffer from low size selectivity. Microfiltration (MF) range pore size of ENMs makes them efficient in removing relatively coarse particles and suspended solids but not tiny substances smaller than the pore size..$^{3-7}$ Considering the importance of separation of nanoparticles also organics that can be detrimental to the quality of water systems, optimizing the selectivity of such membranes could be crucial. Accordingly, not only the high permeability of the membrane is preserved but also on the basis of selectivity, the application domain would be extended from MF to ultrafiltration (UF) and even nanofiltration. Long-term functionality, that is, longevity of ENMs with regard to their extraordinary surface area thereby a higher exposed surface to the water streams is dependent on their mechanical stability. Hence, to maximize the efficiency of an ENM, besides the optimization of the selectivity, its mechanical stabilization should be also stressed.

Here, we show that through one-single approach, that is, protein functionalization, an ENM can acquire mechanical and thermal stability while showing more optimum selectivity. Inspired by the

*Corresponding author e-mail addresses: mady.elbahri@hzg.de; me@tf.uni-kiel.de 
Bioinspired, Biomimetic and Nanobiomaterials Volume 2 Issue BBN4
Biofunctionalized nanofibrous membranes

mimicking carnivorous plants

Homaeigohar, Disci-Zayed, Dai and Elbahri nature, similar to a carnivorous plant, which spreads the leaves equipped with some sticky mucilage to attract and capture insects, here, we show that through protein functionalization, that is, immobilization, an ENM can acquire more optimum selectivity in an analogous way. Thanks to water-protein interaction, subsequent conformational change and protein-metal and protein-protein interactions, the protein immobilized onto the nanofibers is assumed to capture not only very small nanoparticles ${ }^{8}$ but also tiny biomolecules normally passing through the pores leading to a higher selectivity. This behavior brings about a breakthrough in applicability of the ENMs and extends the application area beyond MF to UF. This approach through cross-linking of the nanofibers could be positively influential on mechanical stability of the membrane as well. As another promising outcome, decoration of ENMs with metal nanoparticles filtered out from the respective aqueous suspension would give rise to a nanohybrid with synergetic optical, electrical, catalytic and biological properties. ${ }^{9}$ Thus, for the first time, we also report a filtration-based approach for fabrication of a nanohybrid with well-dispersed nanoparticles applicable for a naked eye plasmonic detection of protein denaturation/renaturation.

\section{Experimental}

\subsection{Materials}

Poly(acrylonitrile-co-glycidyl methacrylate) (PANGMA) as the polymer to be electrospun and to be formed as nanofibers was synthesized at Helmholtz-Zentrum Geesthacht (Geesthacht, Germany; previously GKSS Forschungszentrum $\mathrm{GmbH})^{10}$ with a molecular weight $(\mathrm{Mn})$ of ca. $100,000 \mathrm{~g} / \mathrm{mol}$ and glycidyl methacrylate (GMA) content of $13 \mathrm{~mol} \%$. Bovine serum albumin (BSA; dried powder) and phosphate-buffered saline (PBS) were purchased from Sigma-Aldrich Co., (USA). N,N-dimethylformamide (DMF) was obtained from Merck (Germany). As the model for the nanoparticles to be filtered out, the Au nanoparticles of $40 \mathrm{~nm}$ were made by sodium citrate reduction method. ${ }^{11}$

\subsection{Electrospinning and biofunctionalization of PANGMA nanofibers}

DMF was used to dissolve PANGMA (20 wt.\%). The solution was fed with a constant rate of $1.1 \mathrm{ml} / \mathrm{h}$ into a needle by using a syringe pump (Harvard Apparatus, USA). The electrospinning was performed using aluminum (Al) foil located $25 \mathrm{~cm}$ above the needle tip for $4 \mathrm{~h}$ by applying a voltage of $15-20 \mathrm{kV}$ (Heinzinger Electronic GmbH, Germany). After all, the nanofibrous mat was peeled off from $\mathrm{Al}$ foil and dried under vacuum at $30^{\circ} \mathrm{C}$ for $24 \mathrm{~h}$ to remove the residual solvent.

In the next step, the PANGMA nanofibrous mats were immersed into the BSA/PBS buffer $(5 \mathrm{mg} / \mathrm{ml}$; $\mathrm{pH} 6 \cdot 8)$ while gently shaken at $55^{\circ} \mathrm{C}$ for $24 \mathrm{~h}$. Subsequently, to remove all the unbound BSA, the membranes were taken out and washed several times by PBS buffer $(\mathrm{pH} \mathrm{6.8)}$ and then deionized water and dried under vacuum at $30^{\circ} \mathrm{C}$ for $24 \mathrm{~h}$

\subsection{Characterization of structural properties}

The morphology of the PANGMA ENMs as neat and biofunctionalized was observed with a scanning electron microscope (SEM; LEO Gemini 1550 VP, Zeiss) at $10 \mathrm{kV}$ accelerating voltage after gold coating. The diameter of the electrospun nanofibers was determined from the SEM images using the Adobe Acrobat v.07 software.

To investigate the eventual influence of the biofunctionalization on thermal and mechanical properties of the membranes, differential scanning calorimetry (DSC), thermal gravimetric analysis (TGA) and tensile tests were performed. DSC was performed by a Netzsch DSC 204 Phoenix ${ }^{\circledR}$ using indium standards. The glass-transition temperature $\left(T_{\mathrm{g}}\right)$ was determined by means of a dynamic scan at $10^{\circ} \mathrm{C} / \mathrm{min}$ from 20 to $300^{\circ} \mathrm{C}$. The thermal stability of the neat and biofunctionalized ENMs was evaluated by TGA. TGA of the ENMs was carried out with a thermogravimetric analyzer of Netzsch 209 TG. TGA analysis was performed at $20-700^{\circ} \mathrm{C}$ with a heating rate of $10^{\circ} \mathrm{C} / \mathrm{min}$ under argon. The decomposition temperature $\left(T_{\mathrm{d}}\right)$ was defined as the temperature at $5 \%$ weight loss.

The ENMs' mechanical properties were also characterized by a tensile machine (Zwick/Roell Z020-20KN, Germany) equipped with a $20 \mathrm{~N}$ load cell at ambient temperature. The cross-head speed was $2 \mathrm{~mm} / \mathrm{min}$ and the gauge length was $20 \mathrm{~mm}$. The reported tensile moduli and tensile strengths represent average results of ten tests.

\subsection{Characterization of filtration properties \\ 2.4.1 Filtration of protein}

The ability of the biofunctionalized ENMs in removing proteins from water was investigated via a filtration experiment of BSA.

To run the experiment, $20 \mathrm{ml}$ protein aqueous solution $(2 \mathrm{mg} / \mathrm{l})$ was passed through the membranes with an active filtration area of $2 \mathrm{~cm}^{2}$. The driving force for the filtration was a minor hydrostatic pressure of $20 \mathrm{mPa}$ supplied by an installed column on the module. The experiment was performed in a cyclic style, that is, every permeate was considered as the new feed for the new filtration cycle until completion of five successive cycles.

Already constructed the standard protein calibration curve, the concentration of the proteins in the original feed and permeates was specified by a UV-Vis spectroscopy. The filtration efficiency (FE) was determined according to the Equation 1:

1.

$$
\mathrm{FE}=\left(1-\frac{C_{\mathrm{p}}}{C_{\mathrm{f}}}\right) \times 100 \%
$$

where $C_{\mathrm{p}}$ and $C_{\mathrm{f}}$ are the concentrations of protein in the permeates and feeds, respectively. 
Bioinspired, Biomimetic and Nanobiomaterials Volume 2 Issue BBN4
Biofunctionalized nanofibrous membranes

mimicking carnivorous plants

Homaeigohar, Disci-Zayed, Dai and Elbahri

\subsubsection{Filtration of nanoparticles}

The retention capability of the neat and biofunctionalized PANGMA ENMs was determined using aqueous suspensions $(20 \mathrm{ml})$ containing gold nanoparticles of $40 \mathrm{~nm}$. The filtration test was performed in a same manner as the protein filtration experiment. This process was performed in three successive cycles. Noteworthy, the filtration was performed under a negligible hydrostatical pressure, that is, no considerable feed pressure. The retention ability of the ENM for the gold nanoparticles was characterized by UV-Vis spectroscopy.

\section{Results and discussion}

As a new polymeric material to be used for nanofiber production, PANGMA is a copolymer of acrylonitrile (AN) and glycidyl methacrylate GMA. ${ }^{6}$ The main advantages of this polymer include the chemical stability from the sturdy backbone of polyacrylonitrile and the optimum functionality, that is, further reacting ability owing to presence of the free and active epoxy group on GMA. The latter possibility offers the opportunity in a variety of activation/coupling chemistries for the covalent binding of capturers. PANGMA nanofibers were functionalized through surface immobilization with BSA. BSA acting as the capturer component is a cheap serum albumin protein, widely used in numerous biochemical applications.

As shown in Figure 1, the biofunctionalized ENM possesses beadless nanofibers with smooth surface. Moreover, as seen in this figure, the biofunctionalization does not sacrifice the porosity of the mat, that is, clogging the pores. On the basis of the measurement of nanofibers' diameter (Figure 2), the fibers after the biofunctionalization are slightly enlarged in diameter. This growth of nanofibers certainly does not alter the porous structure, that is, pore size of the membranes.

The functionalization of the nanofibers is according to the extremely simple and extensively used epoxy amino reaction between the amine groups on BSA and epoxy groups on PANGMA. ${ }^{6,12}$ Figure 3 shows a schematic sketch of the functionalization process illustrating how BSA ligands can concurrently bond to two adjacent nanofibers, thereby form a chemical cross-linking, which is expected to improve the mechanical and thermal stabilities of the membrane.

Accordingly as seen in Figure 4, the biofunctionalized membranes possess a higher level of thermal resistance. The main reason could be cross-linking of the PANGMA chains stimulated by the functionalization. The cross-linking restricts local chain mobility thereby increases $T_{\mathrm{g}}{ }^{13}$ Moreover, a higher thermal stability is induced by the cross-linking, ${ }^{14}$ implying the feasibility of application of the biofunctionalized membrane at higher temperatures than ambient temperature but certainly lower than denaturation temperature of the BSA.

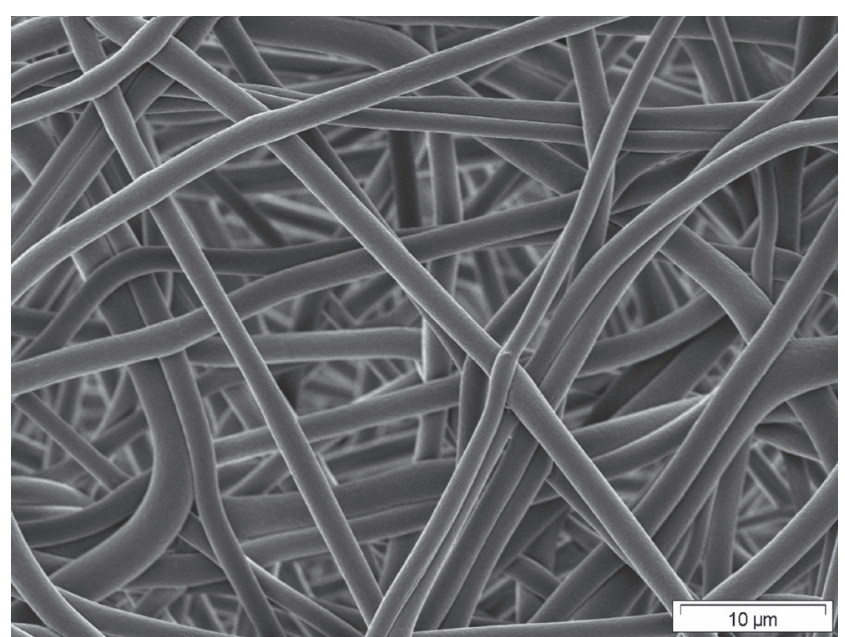

Figure 1. Surface morphology of the PANGMA nanofibers as BSA biofunctionalized. BSA, bovine serum albumin; PANGMA, poly(acrylonitrile-co-glycidyl methacrylate).

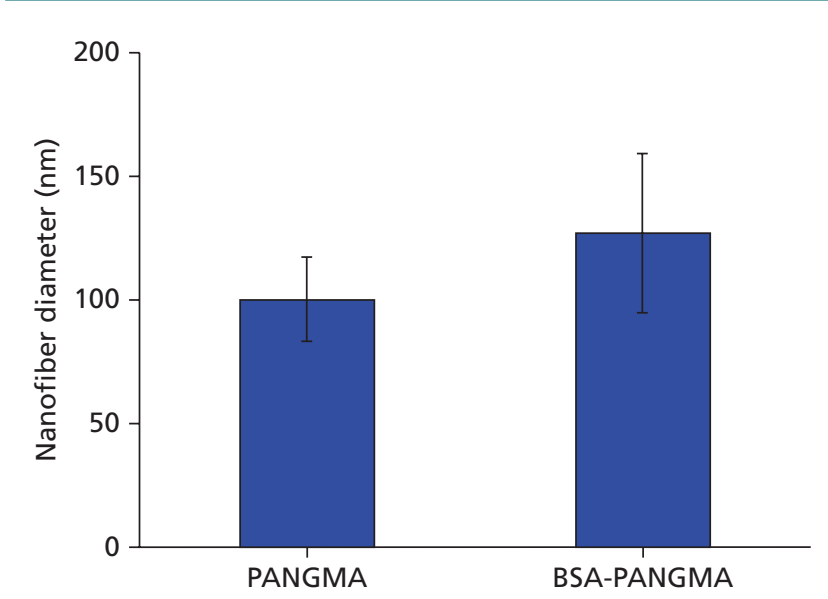

Figure 2. Average diameter of the PANGMA nanofibers as neat and BSA biofunctionalized. BSA, bovine serum albumin; PANGMA, poly(acrylonitrile-co-glycidyl methacrylate).

As a prerequisite for pressure-driven liquid filtration applications, ${ }^{15}$ as seen in Figure 5, the biofunctionalization of the PANGMA ENMs could lead to a more optimum mechanical performance in terms of stiffness (elastic modulus) also strength. As mentioned earlier, BSA ligands act as a cross-linker forming a network structure that restricts movement of the nanofibers tending to pass by each other thereby optimize the mechanical performance of the biofunctionalized membrane.

Applicability of ENMs is revolutionized when they are able to filter out not only coarse suspended solids but also nanosolids and organics much smaller than the pore size. Here, we aim to show that the biofunctionalization approach brings about a high selectivity to nanoparticles also biomolecules. As the model for the filtrates, $\mathrm{Au}$ nanoparticles $(40 \mathrm{~nm})$ and BSA were selected and their respective 
Bioinspired, Biomimetic and Nanobiomaterials Volume 2 Issue BBN4
Biofunctionalized nanofibrous membranes mimicking carnivorous plants

Homaeigohar, Disci-Zayed, Dai and Elbahri
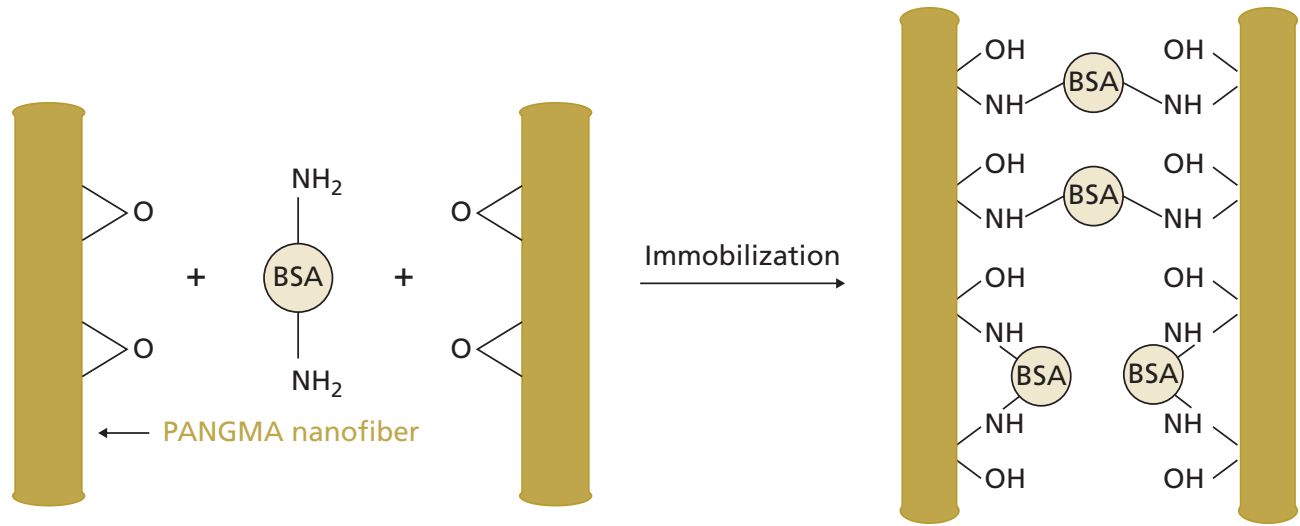

Figure 3. Schematic diagram of the immobilization of BSA onto PANGMA nanofibers. BSA, bovine serum albumin; PANGMA, poly(acrylonitrile-co-glycidyl methacrylate)



Figure 4. Variation of thermal properties of the PANGMA ENMs before and after the biofunctionalization. ENM, electrospun nanofibrous membranes; PANGMA, poly(acrylonitrile-co-glycidyl methacrylate)

suspension and solution, respectively, were passed through the neat and biofunctionalized ENMs.

As shown in Figure 6, based on the UV-Vis graph, in contrary to the neat PANGMA ENM that is able to reject a negligible part of the nanoparticles, the biofunctionalized ENM holds them significantly. Such an efficient removal as adsorption of the nanoparticles to the surface of nanofibers is obviously observed in Figure 7.

The SEM image shown in Figure 7 implies that the metal nanoparticles are arranged uniformly on the biofunctionalized nanofibers. It is assumed that the immobilized protein plays an important role in the uniform dispersion of the gold nanoparticles.

The reason for such an optimum FE by the BSA/PANGMA ENMs is assumed to be $\mathrm{pH}$ - dependent conformational change of

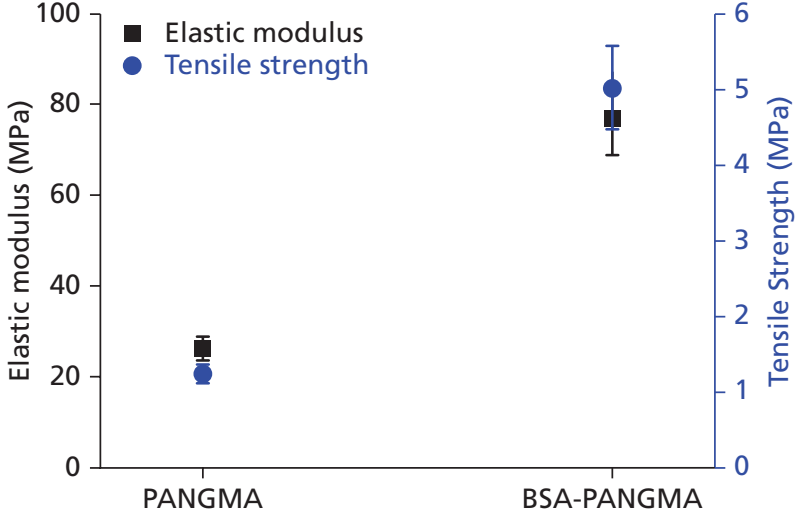

Figure 5. Tensile properties of the PANGMA ENMs before and after the biofunctionalization. ENM, electrospun nanofibrous membranes; PANGMA, poly(acrylonitrile-co-glycidyl methacrylate).

BSA. As soon as wetting, $\mathrm{pH}$ increases to above isoelectric point of BSA, that is, $4 \cdot 7$, therefore the folded state, that is, " $\alpha$ - helix," is unfolded as $\beta$-turn, and tryptophan and cysteine residue groups also amine-based groups which due to strong hydrogen bondings between the peptide groups were less available emerge. This alteration is traceable through attenuated total reflectance-fourier transform infrared measurements (Figure shown at Ref. 8) as replacement of the bands assigned to $\alpha$-helix secondary structure of BSA (1650-1658 $\left.\mathrm{cm}^{-1}\right)$ predominated in dry state with those of turns $\left(1666-1673 \mathrm{~cm}^{-1}\right)^{16,17}$ in wet state. When previously buried peptide bonds and amino acid side chains are exposed and become available for interaction with the aqueous environment, the higher functionality leads to binding considerably more water ${ }^{18}$ thereby a swollen structure. The swollen functional protein on the surface of the nanofibers makes a smaller pore size and more steric hindrance by growing nanofibers. On the other hand, through emergence of the new functional groups upon conformational change, a higher chance of interaction between the 
Bioinspired, Biomimetic and Nanobiomaterials Volume 2 Issue BBN4
Biofunctionalized nanofibrous membranes

mimicking carnivorous plants

Homaeigohar, Disci-Zayed, Dai and Elbahri



Figure 6. Separation of nanoparticles $(40 \mathrm{~nm})$ from water by the biofunctionalized PANGMA nanofibrous membranes (the left and right graphs are about the filtration performance of the neat and biofunctionalized ENMs, respectively). ENM, electrospun nanofibrous membranes; PANGMA, poly(acrylonitrile-co-glycidyl methacrylate)

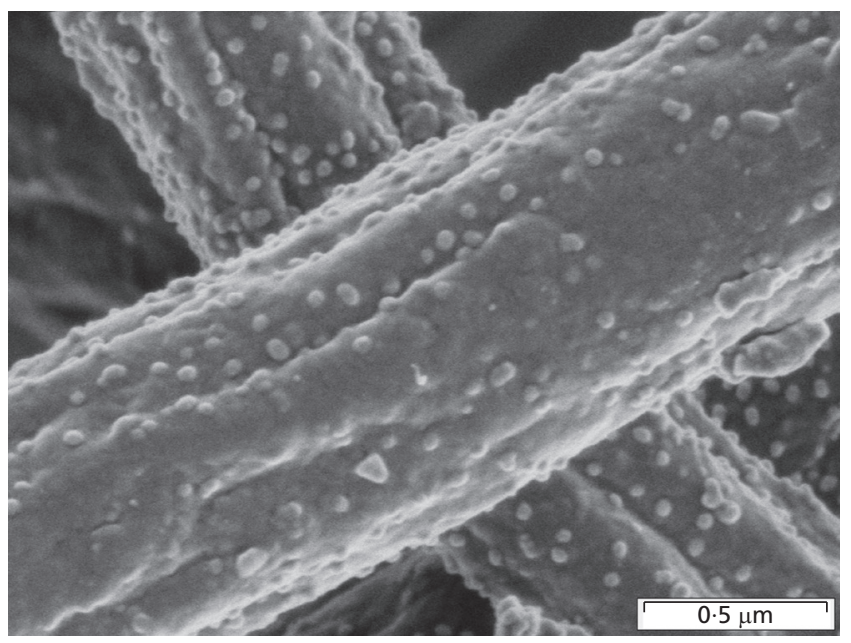

Figure 7. Adsorption of nanoparticles (Au) to the surface of the biofunctionalized PANGMA nanofibers. PANGMA, poly(acrylonitrileco-glycidyl methacrylate).

protein immobilized and the filtrates comes to existence. Strong protein-metal nanoparticle interactions, such as ionic interaction, hydrogen binding, Van der Wals and hydrophilic interactions, ${ }^{19-22}$ make $\mathrm{Au}$ nanoparticles stick to the biofunctionalized nanofiber. Combination of these effects reminds us the hunting behavior of a carnivorous plant and results in a significantly high retention efficiency for the membrane being studied.

Depending on the $\mathrm{pH}$ of the aqueous medium, different behaviors could be observed. When $\mathrm{pH}$ is lowered down to 4 , the conformational change does not occur thereby capturing of the nanoparticles will be suppressed.

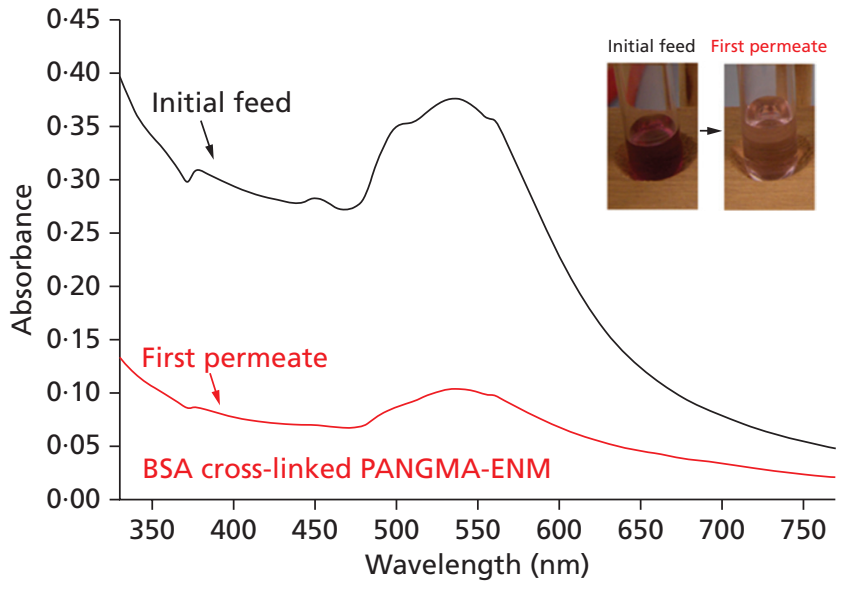

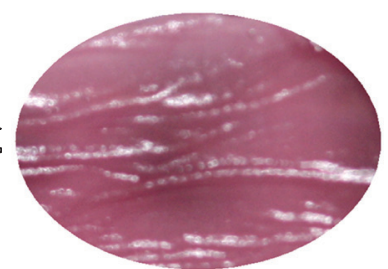

Figure 8. The plasmonic red color of the bionanohybrid at the naturated ( $\mathrm{pH} 7$ - left) and denaturated ( $\mathrm{pH} 3$ - right) states.

It is well known that under highly acidic conditions, that is, a $\mathrm{pH}$ below 4 , the acidic moiety of the protein component is highly protonated, thereby the protein is denaturated. The secondary and tertiary structures of the protein are disrupted leading to an unfolding or straightening effect. These transitions bring about an increased local separation of the domains as the conversion of the compact heart shape of the protein to a linear bead form. Such an expansion could alter the plasmonic state of the nanoparticles stuck to the protein, thereby acting as signal transduction. In the ideal case, this transition should be reversible upon increase of $\mathrm{pH}$, that is, a switchability in plasmonic behavior of the metal nanoparticles. To investigate this switchable plasmonic effect representative of denaturation/renaturation of the protein, the nanohybrid was immersed in an acidic aqueous solution $(\mathrm{pH} 3)$ and subsequently in a neuter aqueous solution ( $\mathrm{pH} 7$ ). As shown in Figure 8, while the plasmonic red color of the bionanohybrid after exposure to the acidic medium is drastically weakened, it is recovered as soon as the $\mathrm{pH}$ rises to 7 in a neuter aqueous medium.

The weak and intensive plasmonic red colors observed at different $\mathrm{pH}$ values represent denaturation and renaturation of the protein, 
Bioinspired, Biomimetic and Nanobiomaterials Volume 2 Issue BBN4
Biofunctionalized nanofibrous membranes mimicking carnivorous plants

Homaeigohar, Disci-Zayed, Dai and Elbahri respectively. This color change occurs owing to coupling/decoupling between the nanoparticles induced by the interparticle distances. Figure 9 schematically illustrates this effect. While in the naturated state, a strong near field coupling between closely spaced nanoparticles occurs, in the denaturated state as the distance between the nanoparticles changes, the effect is diminished. This hypothesis is proved through UV-Vis measurements. Figure 10 shows the reflection spectra of the bionanocomposite in both the states (for a better clarity, only a portion of the full reflectance curves are shown). While the denaturated state is recognized by a single plasmonic dip located at $530 \mathrm{~nm}$, it is obvious that, the intense red color of the naturated membrane shows a wide resonance with a plasmonic shoulder at 572 and a central dip at $523 \mathrm{~nm}$, which is shifted to a lower wavelength compared with the denaturted one. This observations are in good agreement with the expectation from the plasmon hybridization of the individual nanoparticle plasmons. ${ }^{23,24}$ Five successive cycles of exposure of


Figure 9. The sketch illustrates plasmonic coupling between nanoparticles in the naturated state $(\mathrm{pH} 7)$ left and plasmonic decoupling for the denaturated one $(\mathrm{pH} 3)$, right.

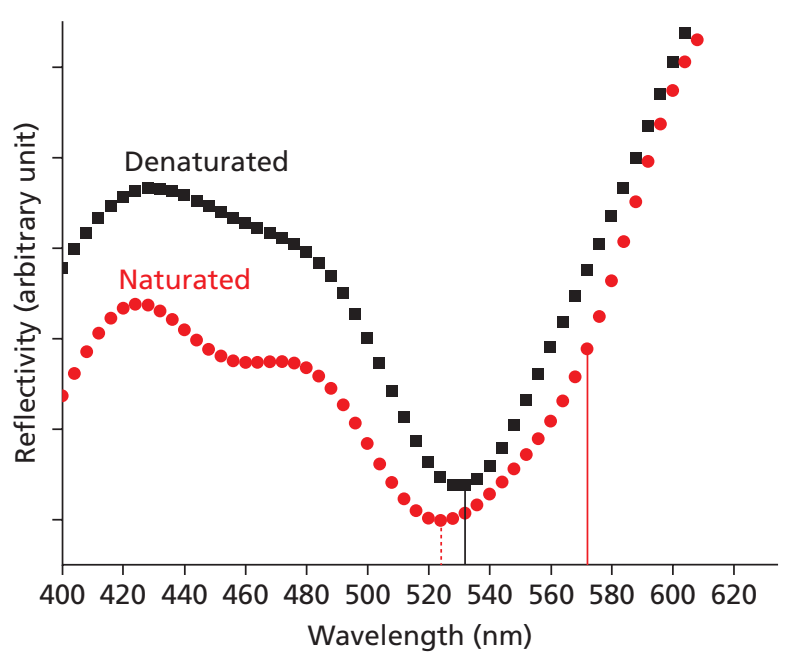

Figure 10. The reflection spectra of the bionanocomposite in the naturated and denaturated states. the bionanohybrid to the aqueous media with different $\mathrm{pH}$ values, verified optimum switchability of the plasmonic color and the spectral shift of the samples.

The promising results about the nanofluid filtration of nanoparticles as small as $40 \mathrm{~nm}$ encouraged us to evaluate the filtration potential of the biofunctionalized ENM for other kind of water pollutants. Therefore, filtration of a protein model such as BSA using the biofunctionalized ENM was performed. Owing to a macroporosity, the nanofibrous membranes can hardly retain soluble substances; however, this study showed that the biofunctionalized ENM is also able to separate a biomolecule such as BSA from water efficiently. As seen in Figure 11, the soluble protein of BSA is optimally separated from water. Surprisingly, after five cycles of continuous filtration, the protein rejection efficiency of the membrane reaches to almost $90 \%$. This value is in contrast to that obtained by the nonfunctionalized ENM. The same principle as that in filtration of metal nanoparticles, that is, the "carnivorous plant" concept is applicable here as well.

The amazing filtration and smart plasmonics properties obtained through the adopted biofunctionalization approach made us optimistic about the high potentials of the carnivorous concept. This is why our research on the concept of biofunctionalized ENMs for filtration of main water pollutants and bridging the filtration area to other advanced technological domains such as plasmonics is going on.

\section{Conclusion}

Biofunctionalization of nanofibers was adopted as an efficient approach for not only optimization of structural properties including thermal and mechanical ones but also water filtration selectivity. The protein immobilization did not change the size of the nanofibers considerably and the porosity remained unchanged.



Figure 11. Protein filtration efficiency of the biofunctionalized ENM. ENM, electrospun nanofibrous membranes. 
Bioinspired, Biomimetic and Nanobiomaterials Volume 2 Issue BBN4
Biofunctionalized nanofibrous membranes

mimicking carnivorous plants

Homaeigohar, Disci-Zayed, Dai and Elbahri
An operative filtration and selectivity to nanosized and soluble substances while possessing an extraordinary interconnected porosity for the nanofibrous membranes could be amazing potentials for the development of new generation of energy saving but efficient membranes. Moreover, decoration of the nanofibers by the functional filtrates such as metal nanoparticles could bring about a novel approach for the fabrication of smart bionanocomposite sensors, for example, for detection of protein denaturation.

\section{Acknowledgements}

M Elbahri thanks the initiative and networking fund of the Helmholtz Associations for providing the financial base of the start-up of his research group. The authors gratefully acknowledge the partial financial support by the German Research Foundation (DFG) through the projects SFB 677 C9. Stefan Rehders for drawing the sketches, and Dr. Rania Khalil and Ramzy Abdelaziz for their valuable cooperation in the experimental part are also acknowledged. The authors would like also to appreciate Silvio Neumann for TGA and DSC measurements, Heinrich Böttcher for tensile tests and Karen-Marita Prause for SEM measurements.

\section{REFERENCES}

1. Thavasi, V.; Singh, G.; Ramakrishna, S. Electrospun nanofibers in energy and environmental applications. Energy \& Environmental Science 2008, 1(2), 205-221.

2. Ramakrishna, S.; Jose, R.; Archana, P. S.; Nair, A. S.; Balamurugan, R.; Venugopal, J.; Teo, W. E. Science and engineering of electrospun nanofibers for advances in clean energy, water filtration, and regenerative medicine. Journal of Materials Science 2010, 45(23), 6283-6312.

3. Aussawasathien, D.; Teerawattananon, C.; Vongachariya, A. Separation of micron to sub-micron particles from water: electrospun nylon-6 nanofibrous membranes as pre-filters. Journal of Membrane Science 2008, 315(1-2), 11-19.

4. Gopal, R.; Kaur, S.; Ma, Z. W.; Chan, C.; Ramakrishna, S.; Matsuura, T. Electrospun nanofibrous filtration membrane. Journal of Membrane Science 2006, 281(1-2), 581-586.

5. Gopal, R.; Kaur, S.; Feng, C. Y.; Chan, C.; Ramakrishna, S.; Tabe, S.; Matsuura, T. Electrospun nanofibrous polysulfone membranes as pre-filters: particulate removal. Journal of Membrane Science 2007, 289(1-2), 210-219.

6. Godjevargova, T.; Konsulov, V.; Dimov, A. Preparation of an ultrafiltration membrane from the copolymer of acrylonitrileglycidylmethacrylate utilized for immobilization of glucose oxidase. Journal of Membrane Science 1999, 152(2), 235-240.

7. Homaeigohar, S. S. H.; Buhr, K.; Ebert, K. Polyethersulfone electrsopun nanofibrous composite membrane for liquid filtration. Journal of Membrane Science 2010, 365, 68-77.
8. Elbahri, M.; Homaeigohar, S.; Dai, T.; Abdelaziz, R.; Khalil, R.; Zillohu, A. U. Smart metal-polymer bionanocomposites as omnidirectional plasmonic black absorbers formed by nanofluid filtration. Advanced Functional Materials 2012, 22(22), 4771-4777.

9. Faupel, F.; Zaporojtchenko, V.; Strunskus, T.; Elbahri, M. Metal-polymer nanocomposites for functional applications. Advanced Engineering Materials 2010, 12(12), 1177-1190.

10. Hicke, H. G.; Lehmann, I.; Malsch, G.; Ulbricht, M.; Becker, M. Preparation and characterization of a novel solvent-resistant and autoclavable polymer membrane. Journal of Membrane Science 2002, 198(2), 187-196.

11. Liu, S. P.; Yang, Z.; Liu, Z. F.; Liu, J. T.; Shi, Y. Resonance Rayleigh scattering study on the interaction of gold nanoparticles with berberine hydrochloride and its analytical application. Analytica Chimica Acta 2006, 572(2), 283-289.

12. Dai, T.; Miletic, N.; Loos, K.; Elbahri, M.; Abetz, V. Electrospinning of poly[acrylonitrile-co-(glycidyl methacrylate)] nanofibrous mats for the immobilization of candida antarctica lipase B. Macromolecular Chemistry and Physics 2010, 212(4), 319-327.

13. Krumova, M., Lopez, D., Benavente, R., Mijangos, C., Perena, J. M. Effect of crosslinking on the mechanical and thermal properties of poly(vinyl alcohol). Polymer 2000, 41(26), 9265-9272.

14. Marechal, E. Chemical modification of synthetic polymers. In Comprehensive Polymer Science (Eastmond, G. C.; Ledwith, A.; Russo, S.; Sigwalt, P. (eds.)). Oxford: Pergamon, 1989, 1-47.

15. Homaeigohar, S. S. H.; Elbahri, M. Novel compaction resistant and ductile nanocomposite nanofibrous microfiltration membranes. Journal of Colloid and Interface Science 2012, 372, 6-15.

16. Ferrer, E. G.; Bosch, A.; Yantorno, O.; Baran, E. J. A spectroscopy approach for the study of the interactions of bioactive vanadium species with bovine serum albumin. Bioorganic \& Medicinal Chemistry 2008, 16(7), 3878-3886.

17. El-Sherif, H.; El-Masry, M.; Abou Taleb, M. F. pH-sensitive hydrogels based on bovine serum albumin for anticancer drug delivery. Journal of Applied Polymer Science 2009, 115(4), 2050-2059.

18. Chou, D. H.; Morr, C. V. Protein-water interactions and functional properties. Journal of the American Oil Chemists' Society 1979, 56(1), A53-A62.

19. Lynch, I.; Dawson, K. A. Protein-nanoparticle interactions. Nano Today 2008, 3(1-2), 40-47.

20. Cedervall, T.; Lynch, I.; Lindman, S.; Berggard, T.; Thulin, E.; Nilsson, H.; Dawson, K. A.; Linse, S. Understanding the nanoparticle-protein corona using methods to quantify exchange rates and affinities of proteins for nanoparticles. PNAS 2007, 104, 2050-2055. 
Bioinspired, Biomimetic and Nanobiomaterials Volume 2 Issue BBN4
Biofunctionalized nanofibrous membranes mimicking carnivorous plants

Homaeigohar, Disci-Zayed, Dai and Elbahri
21. Kong, X. L.; Huang, L. C.; Hsu, C. M.; Chen, W. H.; Han, C. C.; Chang, H. C. High-affinity capture of proteins by diamond nanoparticles for mass spectrometric analysis. Analytical Chemistry 2005, 77(1), 259-265.

22. Brewer, S. H.; Glomm, W. R.; Johnson, M. C.; Knag, M. K.; Franzen, S. Probing BSA binding to citrate-coated gold nanoparticles and surfaces. Langmuir 2005, 21(20), 9303-9307.
23. Zhang, S.; Bao, K.; Halas, N. J.; Xu, H.; Nordlander, P. Substrateinduced Fano resonances of a plasmonic nanocube: a route to increased-sensitivity localized surface plasmon resonance sensors revealed. Nano Letter 2011, 11(4), 1657-1663.

24. Sheikholeslami, S.; Jun, Y. W.; Jain, P. K.; Alivisatos, A. P. Coupling of optical resonances in a compositionally asymmetric plasmonic nanoparticle dimer. Nano Letter 2010, 10(7), 2655-2660.

\section{WHAT DO YOU THINK?}

To discuss this paper, please email up to 500 words to the managing editor at bbn@icepublishing.com

Your contribution will be forwarded to the author(s) for a reply and, if considered appropriate by the editor-inchief, will be published as a discussion in a future issue of the journal.

ICE Science journals rely entirely on contributions sent in by professionals, academics and students coming from the field of materials science and engineering. Articles should be within 5000-7000 words long (short communications and opinion articles should be within 2000 words long), with adequate illustrations and references. To access our author guidelines and how to submit your paper, please refer to the journal website at www.icevirtuallibrary.com/bbn 